\title{
Advances in Research on the circRNA-miRNA-mRNA regulatory network in lung adenocarcinoma by analysis of microarray data
}

\section{Qing Yao}

Shihezi University

\section{Yong-Lai He}

Department of Emergency, Jinshan Branch Shanghai Sixth People's Hospital Affiliated to Shanghai Jiaotong University, Shanghai 200233.

\section{Li-Juan Pang}

Department of Pathology, Shihezi University School of Medicine \& the First Affiliated Hospital to Shihezi University School of Medicine, Shihezi 832002, Xinjiang, China.

\section{Ning Wang}

Department of Pathology, Shihezi University School of Medicine \& the First Affiliated Hospital to Shihezi University School of Medicine, Shihezi 832002, Xinjiang, China.

\section{Shuang-Shuang Dong}

Department of Pathology, Shihezi University School of Medicine \& the First Affiliated Hospital to Shihezi University School of Medicine, Shihezi 832002, Xinjiang, China.

\section{Mei Er Tu He Ta Mi Shi}

Department of Pathology, Shihezi University School of Medicine \& the First Affiliated Hospital to Shihezi University School of Medicine, Shihezi 832002, Xinjiang, China.

\section{Xiao Feng}

Department of Pathology, Shihezi University School of Medicine \& the First Affiliated Hospital to Shihezi University School of Medicine, Shihezi 832002, Xinjiang, China.

\section{Wen-Hu Zhou}

Xiangya School of Pharmaceutical Sciences, Central South University, Changsha, Hunan, 410013, China

\section{Yan Qi ( $\square$ qiyanyan-2004@126.com )}

Department of Pathology, Shihezi University School of Medicine \& the First Affiliated Hospital to Shihezi University School of Medicine, Shihezi 832002, Xinjiang, China, North 2 road, Shihezi, Xinjiang 832002, P.R. China

\section{Research}

Keywords: Lung adenocarcinoma, circRNA, ceRNA, GEO, TCGA

Posted Date: May 29th, 2020

DOI: https://doi.org/10.21203/rs.3.rs-31056/v1

License: @ (i) This work is licensed under a Creative Commons Attribution 4.0 International License. Read Full License 


\section{Abstract}

Background: The present researches indicate that circular RNAs (circRNAs) play pivotal roles in the generation and development of human cancers. However, function of circRNAs in lung adenocarcinoma is still unknown. Herein, we focused our study on the regulation mechanism of circRNAs in lung adenocarcinoma (LUAC)

Methods: CircRNAs-related data can be downloaded from Gene Expression Omnibus(GEO) microarray databases. Relevant expression data of miRNAs and mRNAs were obtained from The Cancer Genome Atlas (TCGA) databases. The differentially expressed circRNAs (DEcircRNAs) were obtained by the robust rank aggregation method, and constructed a ceRNA network with circRNA-miRNA pairs and miRNA-mRNA pairs. The function and pathway enrichment of different genes were analyzed, and the protein-protein interaction (PPI) was predicted by String software, and the subnetwork management module was constructed by MCODE plugin. The differentially expressed mRNAs (DEmRNAs) were used to establish survival analysis by R software.

Results: A total of 19 up-regulated circRNAs, 45 down-regulated circRNAs, 98 up-regulated miRNAs, 18 down-regulated miRNAs, 2002 up-regulated mRNAs, and 553 down-regulated mRNAs were obtained in lung adenocarcinoma. The circRNA-miRNA-mRNA network was constructed by six circRNAs (hsa_circ_0003528, hsa_circ_0004315, hsa_circ_0005699, hsa_circ_0002588, hsa_circ_0005777, hsa_circ_0027033), 19 miRNAs and 33 mRNAs. GO and KEGG pathway analysis indicated that DEmRNAs might be related to the progression of LUAC. The PPI network was constructed and four hub genes (CEP55, CHEK1, CDC25A, KIF23) were obtained from the network, and four hub genes were found to be associated with the same miRNA and circRNA, including hsa_circ_0004315/hsa-miR-195/CEP55, CHEK1, CDC25A, KIF23. Three hub genes (CEP55, CHEK1, KIF23) were significantly associated with LUAC survival and prognosis, and the overall survival rate of lung adenocarcinoma was decreased in high-expression genes compared with low-expression genes.

Conclusion: Our finding indicated that circRNA-miRNA- mRNA axis paly key role in the pathogenesis of lung adenocarcinoma, and some circRNAs could be considered as a biomarker and target for the diagnosis and therapy of LUAC patients.

\section{Background}

Lung cancer is one of the most common disease of cancer-related death in the past ten years (2008-2017) [1], and the five-year survival rate for patients with lung cancer is only $19 \%$. Lung adenocarcinoma (LUAC) accounts for $40 \%$ of lung cancer types, with more than 1 million deaths annually worldwide [2, 3]. In recent decades, circRNAs have great potential as prognostic and diagnostic biomarkers for LUAC [4].

Circular RNAs (circRNAs) contain a large number of non-coding RNAs, which are generated for non-canonical splicing events [5], have high stability because circRNAs are closed circular structures [6], and circRNAs have the function of acting as microRNA (miRNA) sponges to regulate gene expression [7]. Some studies have shown that circRNAs exist stably in some tissues, especially cancer [8], including LUAC [9]. Thus, circRNAs have become a new hot topic in cancer. Xu Y et al. [10] showed that high expression of hsa_ circ_0000326 was related to tumor size, regional lymph node status and differentiation of human lung adenocarcinoma. hsa_circ_0000326 acts as a sponge for miR-338-3p and changes the function of miR-338-3p, which in turn upregulates the expression of downstream target RAB14 and affects the proliferation, migration and apoptosis of lung adenocarcinoma cells. However, mechanisms circRNAs are still unclear in the process of LUAC. 
In this study, the expression profiles of circRNA were obtained from the GEO database and miRNAs and mRNAs were obtained from the TCGA database in LUAC tissues and adjacent normal tissues. Differentially expressed circRNA, miRNA, and mRNA were identified by a robust rank aggregation method. Construction of ceRNA network with circRNAmiRNA pairs and miRNA-mRNA pairs. Through functional enrichment of gene ontology (GO) and Kyoto Encyclopedia of Genes and Genomes (KEGG), the functional pathway of mRNA in this network was analyzed. We then established a protein-protein interaction (PPI) network and extracted hub genes from the PPI network. To better understand the pathogenesis of LUAC, we also constructed a circRNA-miRNA-hub gene subnetwork regulation module and carried out a survival analysis of the hub gene. The flow chart of research ideas is presented in Fig. 1.

\section{Material And Methods}

\section{Data acquisition}

GEO (http://www.ncbi.nlm.nih.gov/GEO) is a public functional genome database. CircRNA microarray data were obtained from GSE101684 and GSE112214 data in the GEO database. Sequencing data of miRNA and RNA were downloaded from the TCGA data portal (https://tcga-data.nci.nih.gov/tcga/). The miRNA sequencing data included 483 lung adenocarcinoma tissues and 45 adjacent normal lung adenocarcinoma tissues, and the mRNA sequencing data included 497 lung adenocarcinoma tissues and 54 adjacent normal lung adenocarcinoma tissues. It is not necessary to obtain approval from the Ethics Committee because all data were obtained from public databases (GEO and TCGA).

\section{Acquisition of DEGs}

The raw data were normalized and log2 transformed. Differentially circRNAs (DEcircRNAs) were obtained by the Limma package in normal samples and tumor samples. Then, all DEGs were integrated and ranked using the robust rank aggregation method [11]. Differentially expressed miRNAs (DEmiRNAs)and mRNAs (DEmRNAs) were obtained with edgeR software package, with Log [fold change] $>2$ and adj.P value $<0.01$.

\section{Prediction of miRNA binding sites}

Cancer-Specific CircRNA (CSCD) (https://gb.whu.edu.cn/CSCD/) was used to predict binding sites of miRNA (MREs) [12]. The miRNAs obtained from DEcircRNA in CSCD are considered potential targets for miRNA. These targeted miRNAs were further intersected with the DEmiRNAs from The Cancer Genome Atlas (TCGA).

\section{Predictions of miRNA target gene}

TargetScan, miRTarBase, and miRDB databases were used to predict the connections between miRNAs and mRNAs [13-15]. Only those three databases that identified the mRNAs were considered candidate mRNAs. The final DEmRNAs were obtained from the intersection of candidate genes and DEmRNAs obtained from the TCGA database.

\section{Construction of the ceRNA network}

The circRNA-miRNA pairs and miRNA-mRNA pairs were constructed to circRNA-miRNA-mRNA regulatory networks. Finally, the ceRNA network was established by CytoCope 3.7.2.

\section{GO and KEGG functional enrichment analysis}

gene ontology (GO) is the main tool for annotating genes and analyzing their biological processes (BPs), molecular functions (MFs), and cellular components (CCs). To investigate the main functional pathway of LUAC, DEmRNAs in 
the network were evaluated through GO annotation and KEGG pathway with the cluster profile package in R. P-value $<0.01$ was considered a statistically significant difference.

\section{Construction and module analysis of the PPI network}

The String online database (https://string-db.org/) was used to build the PPI network by DEmRNAs. The Molecular Complex Detection (MCODE) app was used to identify hub genes from the PPI network [16].

\section{Survival analysis}

Kaplan Meier curve was used to analyze the total survival rate of hub genes selected from the PPI network. The logrank test was used for statistical analysis. The threshold for survival prognosis significance was a P-value $<0.05$. A subnetwork was constructed based on based on mRNAs that were meaningful for overall survival.

\section{Results}

\section{Acquisition of DEGs in LUAC}

The expression of circRNAs in LUAC and control tissues was evaluated in two microarray datasets (GSE101684, GSE112214), and the basic information is listed in Table 1. In the circRNA expression profile data, a total of 64 DEcircRNAs, 19 up-regulated and 45 down-regulated were obtained from GEO. The DEcircRNA was selected from the up-regulation and down-regulation to select the highest ranked circRNA for analysis. There are a total of 6 DEcircRNAs, three up-regulation (hsa_circ_0003528, hsa_circ_0005699, hsa_circ_0004315) and three downregulations (hsa_circ_0005777, hsa_circ_0027033, hsa_circ_0002588) $(p<0.01)$ (Fig.2). The basic characteristic of the six circRNAs is listed in Table 2. Their basic structure patterns of six circRNAs are shown (Fig.3). A total of 116 DEmiRNAs, 98 upregulated and 18 downregulated, and 2555 DEmRNAs, 2002 upregulated and 553 downregulated, were identified in the TCGA database $(p<0.01, \log F C>2)$.

\section{Construction of the ceRNA network}

To better understand the relationship between circRNA, miRNA, and mRNA, the circRNA-miRNA-mRNA (ceRNA) network was constructed by per DECs. CSCD database was used for the circRNA-miRNA prediction. The six highestranked circRNAs in the GEO database were found to intersect the corresponding miRNAs from the CSCD database with those obtained from TCGA and identified 19 pairs of interacting circRNAs and miRNAs. Then we identified mRNA targeted by these DEmiRNAs in three databases (miRBD, miRTarBase, and TargetScan). These targeted mRNAs were cross-checked with the DEmRNAs retrieved from the TCGA database, including 33 DEmRNAs. Finally, we used six circRNA nodes, 19 miRNA nodes, and 33 mRNA nodes in Cytoscape 3.7.2 to construct a ceRNA network. (Fig4)

\section{Functional and pathway enrichment analysis}

GO analysis revealed that above identified mRNAs were mainly concentrated in 'regulation of cell cycle', 'renal system development', 'DNA damage checkpoint'(BPs); 'nucleus' (CC); 'cyclin-dependent protein serine/threonine kinase regulator activity'(MF) (Fig 5a). KEGG pathway analysis revealed strong enrichment in the 'MicroRNA in cancer' 'PI3KAkt signaling pathway' and 'small cell lung cancer' (Fig 5b).

\section{Construction of the PPI network and hub gene}

In total, 33 nodes and 42 edges were mapped in the PPI network by STRING 
Software (Fig 6a). The MCODE approach in Cytoscape was used to identify hub genes in the PPI network. Using kcore 2, the highest-scoring node was 7.000 to get an important module containing nine nodes and 28 edges were identified. The most connected hub gene are CEP55, CHEK1, CDC25A, and KIF23(Fig 6b). we constructed a circRNAmiRNA-hub gene subnetwork based on four circRNA-miRNA-gene regulatory modules (hsa_circ0004315/hsa-mir195/CEP55, hsa_circ0004315/hsa-mir-195/CHEK1, hsa_circ0004315/hsa-mir-195/CDC25A, hsa_circ0004315/hsamir-195/KIF23).

\section{Survival analysis of hub gene}

In this study, 522 clinical specimens of lung adenocarcinoma were downloaded from TCGA database. Kaplan-Meier curves were used to analyze the overall survival rate of 33 DEmRNAs in 522 clinical samples, and 13 DEmRNAs were found to be significant for the overall survival analysis $(p<0.05)$. Three of the four hub genes (CEP55, CHEK1, CDC25A, KIF23) obtained from the PPI network were included in 13 DEmRNAs of significance for the overall survival analysis. Three genes (CEP55, CHEK1, KIF23) were most significantly related to the survival and prognosis of patients with $\operatorname{LUAC}(p<0.01)$, and the overall survival rate of lung adenocarcinoma decreased with the high expression gene compared with the low expression gene (Fig. 7).

\section{Discussion}

CircRNAs are highly stable and can be found in exosomes, cell-free saliva, and plasma [17]. So they may become potential biomarkers or therapeutic targets for cancer, including LUCA [18-20]. However, the exact role of circRNAs in LUAC remains largely unknown. To identify whether circRNA plays a role as ceRNA of the LUAC, we performed microarray data analysis to examine DEGs in LUAC samples and normal samples using a robust rank aggregation method. We constructed a circRNA-miRNA-mRNA regulatory network and PPI network based on DEmRNAs. We also constructed a circRNA-miRNA-hub gene subnetwork based on regulatory modules identified in the circRNA-miRNAmRNA network. The hub genes obtained from the PPI network were further analyzed for survival.

Lung adenocarcinoma is prone to metastasis in the early stage, while brain metastasis is a common complication of lung adenocarcinoma, and its incidence increases with the prolongation of survival [21].The most common mutant gene types of lung adenocarcinoma are EGFR, KRAS, etc [22,23], which have corresponding targeted drug therapy, but are prone to drug resistance to targeted drugs. However, a large number of studies have shown that the dysregulation of circRNAs plays a crucial role in the development of LUAC; therefore, further understanding of the biological mechanism of abnormally expressed circRNAs is essential for the discovery of new, promising therapeutic targets for LUAC [24]. YU et al. [25] collected 36 paired LUAC and healthy tissues and concluded that circRNA CMRA was significantly downregulated in cancer tissues, and circRNA cMras overexpression inhibited LUAC growth and metastasis, and its expression was negatively correlated with tumor stage. Liu et al. [26] found that in vitro experiments suggested that hsa_circ_0005962 promoted LUAC cell proliferation.

In current study, six circRNAs (hsa_circ_0003528, hsa_circ_0004315, hsa_circ_0005699, hsa_circ_0002588, hsa_circ_0005777, hsa_circ_0027033) were involved in the ceRNA network. One of these, only hsa_circ_0003528 were studied by Huang Z et al. [27] This study was based on changes in circRNA in plasma samples from 3 patients with active tuberculosis and 3 healthy controls. Then, 61 DEcircRNAs were recorded. And the verification test showed that TB patients have 6 types of circRNA significantly increased in plasma levels, one of which is hsa_circ_0003528. No relevant studies have reported involvement of hsa_circ_0004315, hsa_circ_0005699, hsa_circ_0002588, hsa_circ_0005777, hsa_circ_0027033 in cancer. 
The present study that a total of 33 DEmRNAs and 19 DEmiRNAs were identified and involved in the ceRNA network, some of which have been found as a biomarker for diagnosis and prognosis. To identified the key circRNAs participating in the regulatory network that was established by a PPI network, four genes were identified, including CEP55, CHEK1, CDC25A, KIF23. Previous work has identified these four genes, but there is only one gene linked to miR-195-5p which is CHEK1 in LUAC. Zuo et al [28] found that Upregulation of miR-195 in LUAC cells was found to decrease CHEK1 and increase Bax expression.In addition, the overexpression of miR-195 enhanced the sensitivity of Lac cells to cisplatin, promoted the apoptosis of Lac cells, and inhibited cell proliferation. Fu F et al [29] showed that CEP55 was related to brain metastasis after LUAC operation. Takamochi et al [30] found that LUAC with EGFR mutations were biologically inert. In EGFR mutant LUAC, the cell cycle-related CDC25A gene is specifically downregulated. Iltzsche et al. [31] found that RNA interference-mediated depletion of KIF23 inhibited lung tumor formation in vivo and induced cell apoptosis in lung cancer cell lines. The results indicated that inhibition of KIF23

may be a strategy for the treatment of lung cancer. However, there no report that the linkages between miR-195-5p and CEP55 and CDC25A and KIF23 in LUAC. Herein, we identified four circRNA-miRNA-hub gene axes, indicating competitive regulatory relationships of one circRNAs with the four genes in LUAC, and the overall survival rates of the three hub genes (CEP55, CHEK1, KIF23) were statistically significant. However, given that these results are based solely on bioinformatics models, further in-depth studies are critical to verifying the possible role of these four axes in LUAC.

\section{Conclusions}

We screened differentially expressed circRNAs, miRNAs, and mRNAs from publicly available microarray data to construct a circRNA-related ceRNA network. High expression of three hub (CEP55, CHEK1, KIF23) genes is associated with the survival rate of lung adenocarcinoma. Therefore the circRNA-miRNA-hub genes (hsa_circ0004315/hsa-mir195/CEP55,CHEK1, CDC25A, KIF23) regulatory subnetwork reveals an important circRNAs that might be involved in carcinogenesis, providing new insight into the pathogenesis of LUAC, and deserves further study of potential therapeutic targets.

\section{Abbreviations}

LUAC: lung adenocarcinoma ; GEO: Gene Expression Omnibus; TCGA: The Cancer Genome Atlas; DEGs: differentially expressed genes; PPI: protein-protein interactions; KEGG: Kyoto Encyclopedia of Genes and Genomes; CSCD: CancerSpecific CircRNA

\section{Declarations}

\section{Acknowledgements}

None.

\section{Authors' contributions}

QY, YLH and NW performed the experiments and analyzed the data; YQ, WHZ and LJP designed and supervised the study; SSD, NE and XF provided crucial input for the project; QY and YLH wrote the manuscript. All authors read and approved the final manuscript.

\section{Funding}


This work was supported by grants from the National Natural Science Foundation of China (grant no. 81860471, U1903125), and the International Cooperation Projects of Shihezi University (grant no. GJHZ201710).

\section{Availability of data and materials}

The datasets during and/or analyzed during the current study available from the corresponding author on reasonable request.

\section{Ethics approval and consent to participate}

Not applicable.

\section{Consent for publication}

Not applicable.

\section{Competing interests}

The authors declare that they have no competing interests.

\section{Author details}

${ }^{1}$ Department of Pathology, Shihezi University School of Medicine \& the First Affiliated Hospital to Shihezi University School of Medicine, Shihezi 832002, Xinjiang, China. ${ }^{2}$ Department of Emergency, Jinshan Branch Shanghai Sixth People's Hospital Affiliated to Shanghai Jiaotong University, Shanghai 200233.

${ }^{3}$ Xiangya School of Pharmaceutical Sciences, Central South University, Changsha, Hunan, 410013, China. ${ }^{*}$ Contributed equally

\section{References}

1. Siegel RL, Miller KD, Jemal A: Cancer statistics, 2020. CA Cancer J Clin 2020, 70:7-30.

2. Qiu LM, Sun YH, Chen TT, Chen JJ, Ma HT: STRIP2, a member of the striatin-interacting phosphatase and kinase complex, is implicated in lung adenocarcinoma cell growth and migration. FEBS Open Bio 2020, 10:351-361.

3. Cheng TY, Cramb SM, Baade PD, Youlden DR, Nwogu C, Reid ME: The International Epidemiology of Lung Cancer: Latest Trends, Disparities, and Tumor Characteristics. J Thorac Oncol 2016, 11:1653-1671.

4. Qiu M, Xia W, Chen R, Wang S, Xu Y, Ma Z, Xu W, Zhang E, Wang J, Fang T, et al: The Circular RNA circPRKCI Promotes Tumor Growth in Lung Adenocarcinoma. Cancer Res 2018, 78:2839-2851.

5. Kristensen LS, Andersen MS, Stagsted LVW, Ebbesen KK, Hansen TB, Kjems J: The biogenesis, biology and characterization of circular RNAs. Nat Rev Genet 2019, 20:675-691.

6. Barrett SP, Salzman J: Circular RNAs: analysis, expression and potential functions. Development 2016, 143:18381847.

7. Zheng Q, Bao C, Guo W, Li S, Chen J, Chen B, Luo Y, Lyu D, Li Y, Shi G, et al: Circular RNA profiling reveals an abundant circHIPK3 that regulates cell growth by sponging multiple miRNAs. Nat Commun 2016, 7:11215.

8. Jeck WR, Sorrentino JA, Wang K, Slevin MK, Burd CE, Liu J, Marzluff WF, Sharpless NE: Circular RNAs are abundant, conserved, and associated with ALU repeats. RNA 2013, 19:141-157.

9. Wang C, Tan S, Liu WR, Lei Q, Qiao W, Wu Y, Liu X, Cheng W, Wei YQ, Peng Y, Li W: RNA-Seq profiling of circular RNA in human lung adenocarcinoma and squamous cell carcinoma. Mol Cancer 2019, 18:134. 
10. Xu Y, Yu J, Huang Z, Fu B, Tao Y, Qi X, Mou Y, Hu Y, Wang Y, Cao Y, et al: Circular RNA hsa_circ_0000326 acts as a miR-338-3p sponge to facilitate lung adenocarcinoma progression. J Exp Clin Cancer Res 2020, 39:57.

11. Song ZY, Chao F, Zhuo Z, Ma Z, Li W, Chen G: Identification of hub genes in prostate cancer using robust rank aggregation and weighted gene co-expression network analysis. Aging (Albany NY) 2019, 11:4736-4756.

12. Xia S, Feng J, Chen K, Ma Y, Gong J, Cai F, Jin Y, Gao Y, Xia L, Chang H, et al: CSCD: a database for cancer-specific circular RNAs. Nucleic Acids Res 2018, 46:D925-D929.

13. Fromm B, Billipp T, Peck LE, Johansen M, Tarver JE, King BL, Newcomb JM, Sempere LF, Flatmark K, Hovig E, Peterson KJ: A Uniform System for the Annotation of Vertebrate microRNA Genes and the Evolution of the Human microRNAome. Annu Rev Genet 2015, 49:213-242.

14. Huang HY, Lin YC, Li J, Huang KY, Shrestha S, Hong HC, Tang Y, Chen YG, Jin CN, Yu Y, et al: miRTarBase 2020: updates to the experimentally validated microRNA-target interaction database. Nucleic Acids Res 2020, 48:D148D154.

15. Chen Y, Wang X: miRDB: an online database for prediction of functional microRNA targets. Nucleic Acids Res 2020, 48:D127-D131.

16. Bandettini WP, Kellman P, Mancini C, Booker OJ, Vasu S, Leung SW, Wilson JR, Shanbhag SM, Chen MY, Arai AE: MultiContrast Delayed Enhancement (MCODE) improves detection of subendocardial myocardial infarction by late gadolinium enhancement cardiovascular magnetic resonance: a clinical validation study. J Cardiovasc Magn Reson 2012, 14:83.

17. Bahn JH, Zhang Q, Li F, Chan TM, Lin X, Kim Y, Wong DT, Xiao X: The landscape of microRNA, Piwi-interacting RNA, and circular RNA in human saliva. Clin Chem 2015, 61:221-230.

18. Li Y, Zheng Q, Bao C, Li S, Guo W, Zhao J, Chen D, Gu J, He X, Huang S: Circular RNA is enriched and stable in exosomes: a promising biomarker for cancer diagnosis. Cell Res 2015, 25:981-984.

19. Chen S, Huang V, Xu X, Livingstone J, Soares F, Jeon J, Zeng Y, Hua JT, Petricca J, Guo H, et al: Widespread and Functional RNA Circularization in Localized Prostate Cancer. Cell 2019, 176:831-843 e822.

20. Vo JN, Cieslik M, Zhang Y, Shukla S, Xiao L, Zhang Y, Wu YM, Dhanasekaran SM, Engelke CG, Cao X, et al: The Landscape of Circular RNA in Cancer. Cell 2019, 176:869-881 e813.

21. Fu F, Zhang Y, Gao Z, Zhao Y, Wen Z, Han H, Li Y, Chen H: Development and validation of a five-gene model to predict postoperative brain metastasis in operable lung adenocarcinoma. Int J Cancer 2020.

22. Wang Z, Cheng Y, An T, Gao H, Wang K, Zhou Q, Hu Y, Song Y, Ding C, Peng F, et al: Detection of EGFR mutations in plasma circulating tumour DNA as a selection criterion for first-line gefitinib treatment in patients with advanced lung adenocarcinoma (BENEFIT): a phase 2, single-arm, multicentre clinical trial. Lancet Respir Med 2018, 6:681-690.

23. Skoulidis F, Goldberg ME, Greenawalt DM, Hellmann MD, Awad MM, Gainor JF, Schrock AB, Hartmaier RJ, Trabucco SE, Gay L, et al: STK11/LKB1 Mutations and PD-1 Inhibitor Resistance in KRAS-Mutant Lung Adenocarcinoma. Cancer Discov 2018, 8:822-835.

24. Wang L, Liang Y, Mao Q, Xia W, Chen B, Shen H, Xu L, Jiang F, Dong G: Circular RNA circCRIM1 inhibits invasion and metastasis in lung adenocarcinoma through the microRNA (miR)-182/miR-93-leukemia inhibitory factor receptor pathway. Cancer Sci 2019, 110:2960-2972.

25. Yu C, Tian F, Liu J, Su M, Wu M, Zhu X, Qian W: Circular RNA cMras inhibits lung adenocarcinoma progression via modulating miR-567/PTPRG regulatory pathway. Cell Prolif 2019, 52:e12610.

26. Liu XX, Yang YE, Liu X, Zhang MY, Li R, Yin YH, Qu YQ: A two-circular RNA signature as a noninvasive diagnostic biomarker for lung adenocarcinoma. J Trans/ Med 2019, 17:50. 
27. Huang Z, Su R, Qing C, Peng Y, Luo Q, Li J: Plasma Circular RNAs hsa_circ_0001953 and hsa_circ_0009024 as Diagnostic Biomarkers for Active Tuberculosis. Front Microbio/ 2018, 9:2010.

28. Zuo W, Zhang W, Xu F, Zhou J, Bai W: Long non-coding RNA LINC00485 acts as a microRNA-195 sponge to regulate the chemotherapy sensitivity of lung adenocarcinoma cells to cisplatin by regulating CHEK1. Cancer Cell Int 2019, 19:240.

29. Li B, Cui Y, Diehn M, Li R: Development and Validation of an Individualized Immune Prognostic Signature in EarlyStage Nonsquamous Non-Small Cell Lung Cancer. JAMA Oncol 2017, 3:1529-1537.

30. Takamochi K, Mogushi K, Kawaji H, Imashimizu K, Fukui M, Oh S, Itoh M, Hayashizaki Y, Ko W, Akeboshi M, Suzuki K: Correlation of EGFR or KRAS mutation status with 18F-FDG uptake on PET-CT scan in lung adenocarcinoma. PLoS One 2017, 12:e0175622.

31. Iltzsche F, Simon K, Stopp S, Pattschull G, Francke S, Wolter P, Hauser S, Murphy DJ, Garcia P, Rosenwald A, Gaubatz S: An important role for Myb-MuvB and its target gene KIF23 in a mouse model of lung adenocarcinoma. Oncogene 2017, 36:110-121.

\section{Tables}

Table 1 The basic information of two microarray from GEO

\begin{tabular}{cllllllll} 
Date source & Platform & Organism & Author & Year & Experiment Type & $\begin{array}{l}\text { Sample } \\
\text { size(T/N) }\end{array}$ & $\begin{array}{l}\text { No.of } \\
\text { circRNAs }\end{array}$ \\
\hline GSE101684 & GPL21825 & $\begin{array}{l}\text { Homo } \\
\text { sapiens }\end{array}$ & Xu M & 2019 & $\begin{array}{l}\text { Non-coding RNA } \\
\text { profiling array }\end{array}$ & $4 / 4$ & 9115 \\
\hline GSE112214 & GPL19979 & $\begin{array}{l}\text { Homo } \\
\text { sapiens }\end{array}$ & $\begin{array}{l}\text { Dong } \\
\text { Y }\end{array}$ & 2019 & $\begin{array}{l}\text { Non-coding RNA } \\
\text { profiling array }\end{array}$ & $3 / 3$ & 4408
\end{tabular}

Table 2 Basic information of Differentially Expression circRNAs

\begin{tabular}{cllllll} 
circRNA ID & Position & Strand & $\begin{array}{l}\text { Genomic } \\
\text { length }\end{array}$ & Best transcript & $\begin{array}{l}\text { Gene } \\
\text { Symbol }\end{array}$ & Regulation \\
\hline hsa_circ_0003528 & $\begin{array}{l}\text { chr5:134032815- } \\
134044578\end{array}$ & + & 11763 & NM_021982 & SEC24A & up \\
\hline hsa_circ_0005699 & $\begin{array}{l}\text { chr16:19627435- } \\
19663412\end{array}$ & + & 35977 & NM_020314 & C16orf62 & up \\
\hline hsa_circ_0004315 & $\begin{array}{l}\text { chr16:74491771- } \\
74493687\end{array}$ & - & 1916 & NR_027264 & GLG1 & up \\
\hline hsa_circ_0005777 & $\begin{array}{l}\text { chr5:73136304- } \\
73136585\end{array}$ & + & 281 & NM_001080479 & RGNEF & down \\
\hline hsa_circ_0027033 & $\begin{array}{l}\text { chr12:56962758- } \\
56965639\end{array}$ & + & 2881 & NM_002898 & RBMS2 & down \\
\hline hsa_circ_0002588 & $\begin{array}{l}\text { chr12:56956200- } \\
56965639\end{array}$ & + & 9439 & NM_002898 & RBMS2 & down
\end{tabular}

\section{Figures}




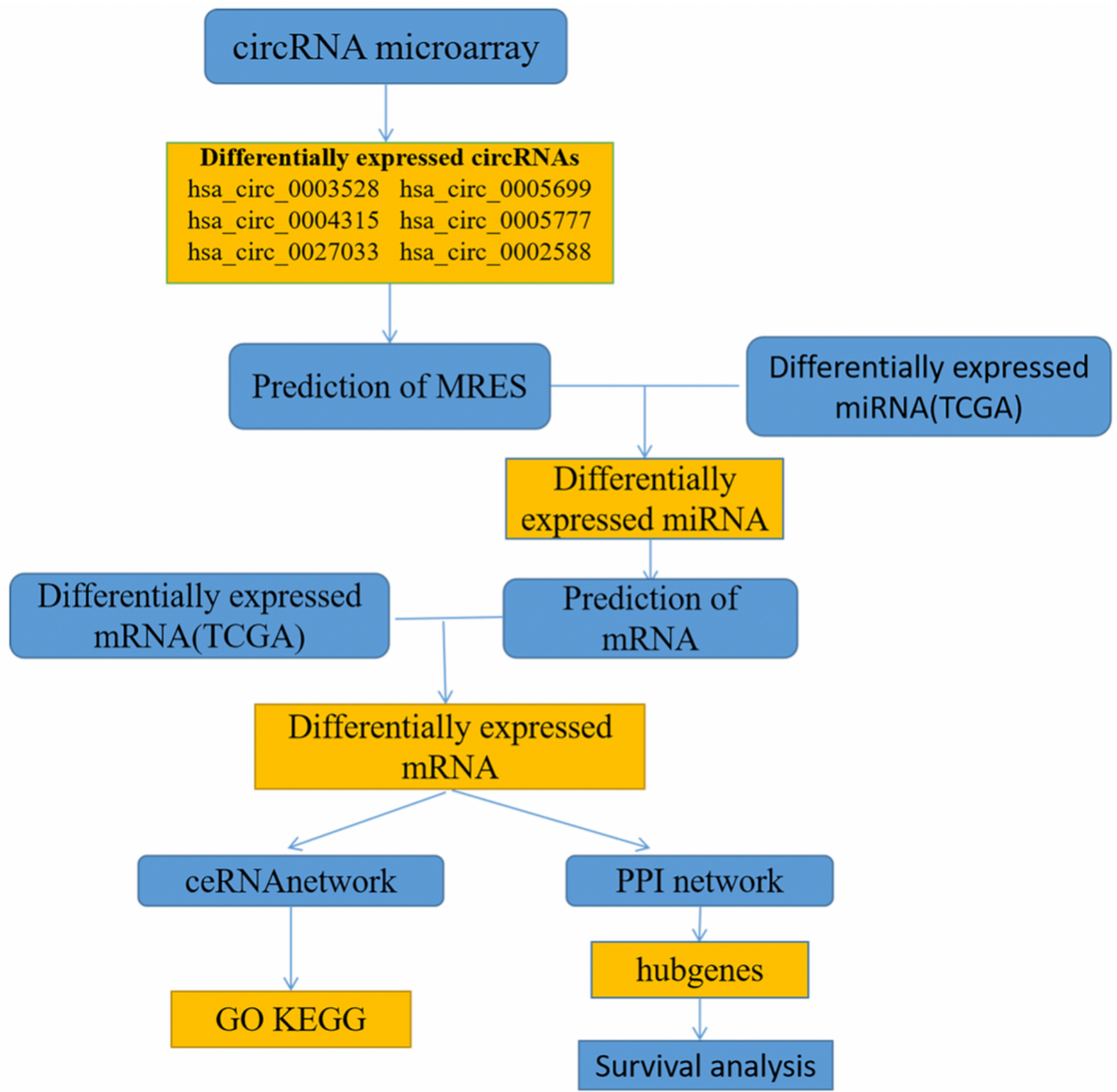

Figure 1

The flow chart of research ideas is presented 


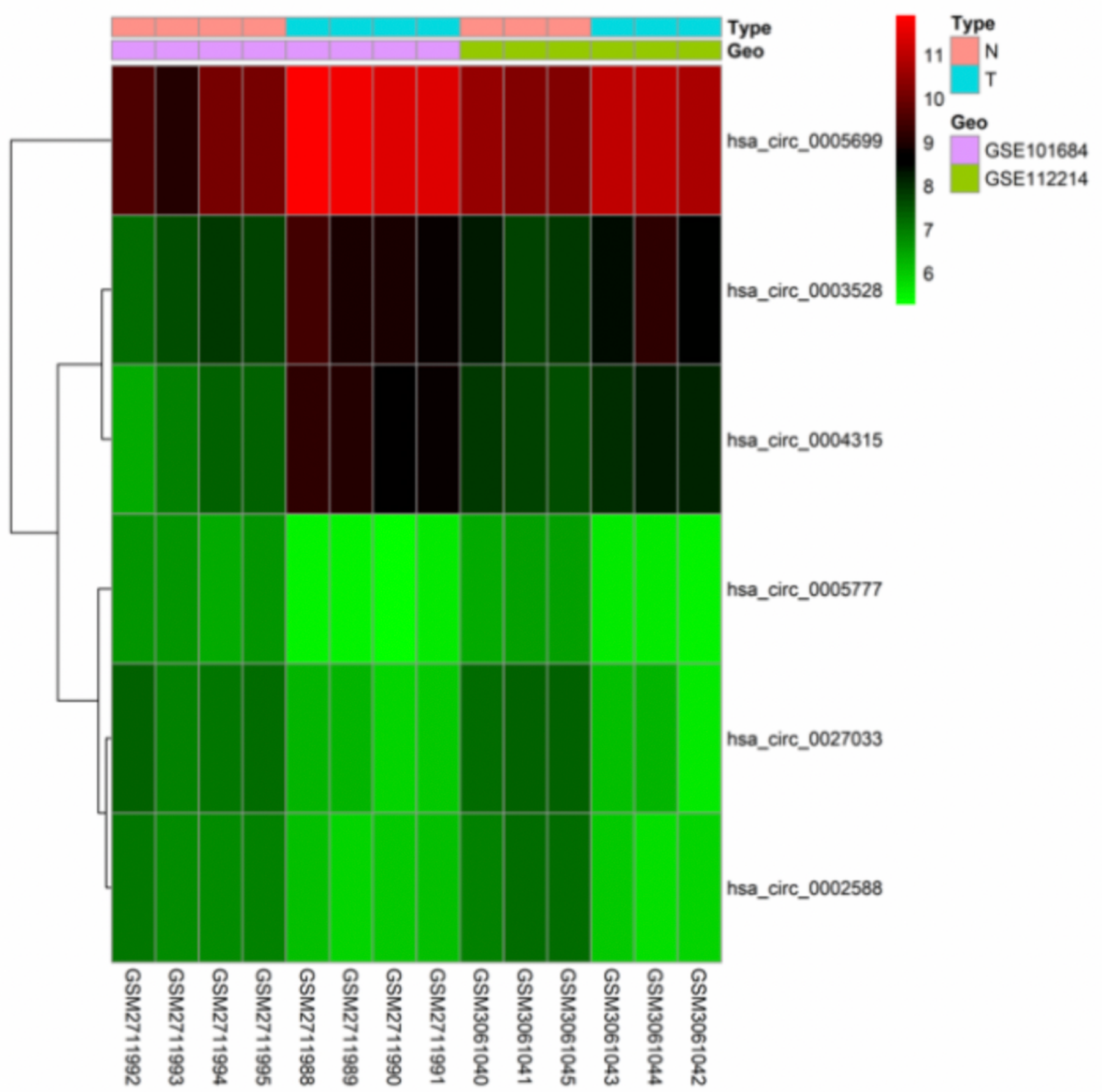

Figure 2

Heatmap of the six differentially expressed circRNAs in the two microarray datasets 
MRE(microRNA response element)

RBP(RNA binding protein)

ORF(open reaing frame)

a

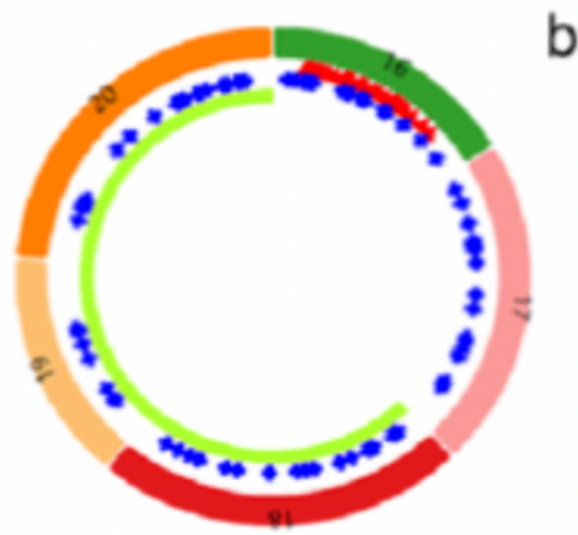

Exonic CircRINA

hsa_circ_0003528

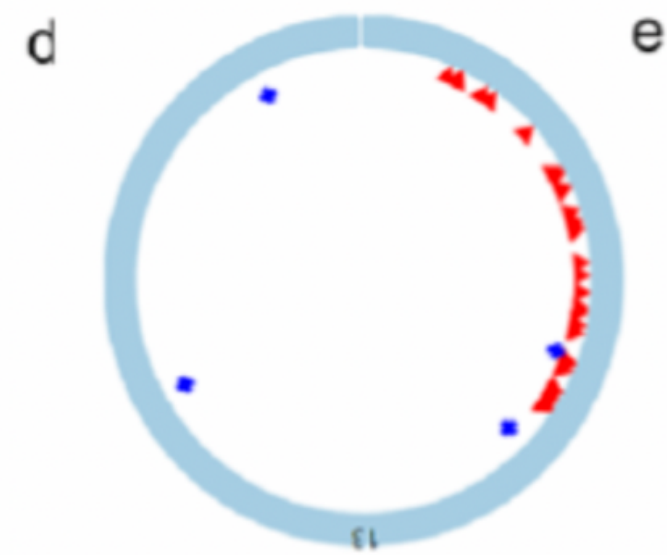

Fxonic CirrRnA hsa_circ_0005777

b

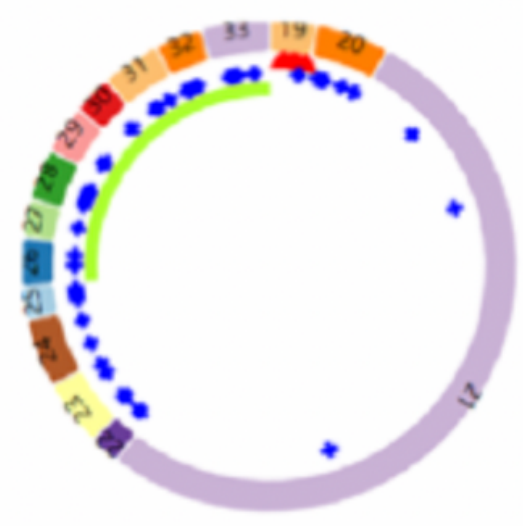

Exonic CircRNA

$$
\text { hsa_circ_0005699 }
$$

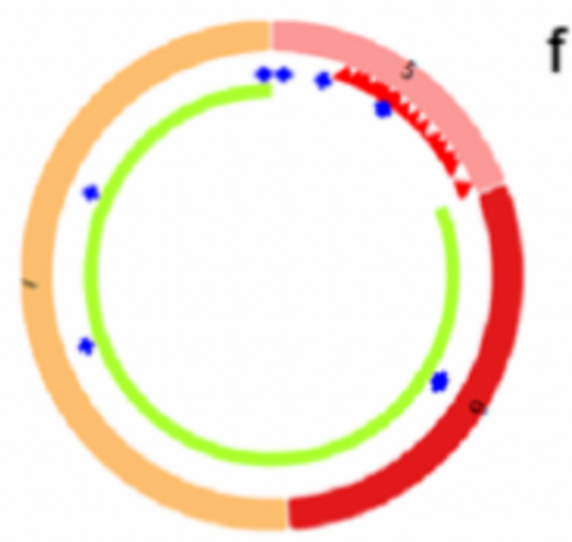

Exonic CircRNA

hsa_circ_0027033

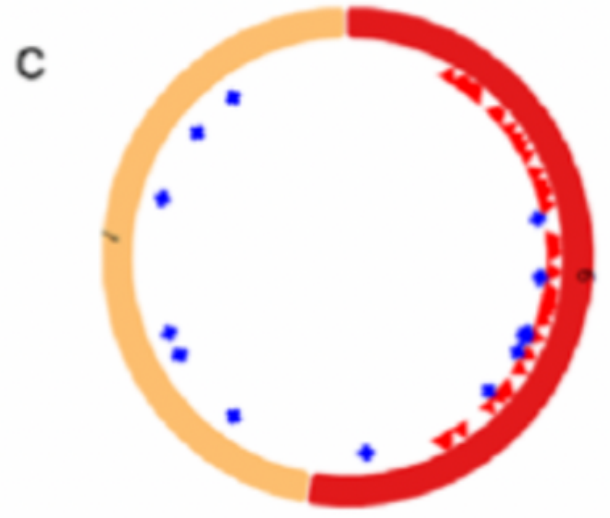

Exonic CircRNe hsa_circ_0004315

\section{Figure 3}

Their basic structure patterns of six circRNAs: a hsa_circ_0003528, b hsa_circ_0005699, c hsa_circ_0004315, d hsa_circ_0005777, e hsa_circ_0027033,f hsa_circ_0002588 


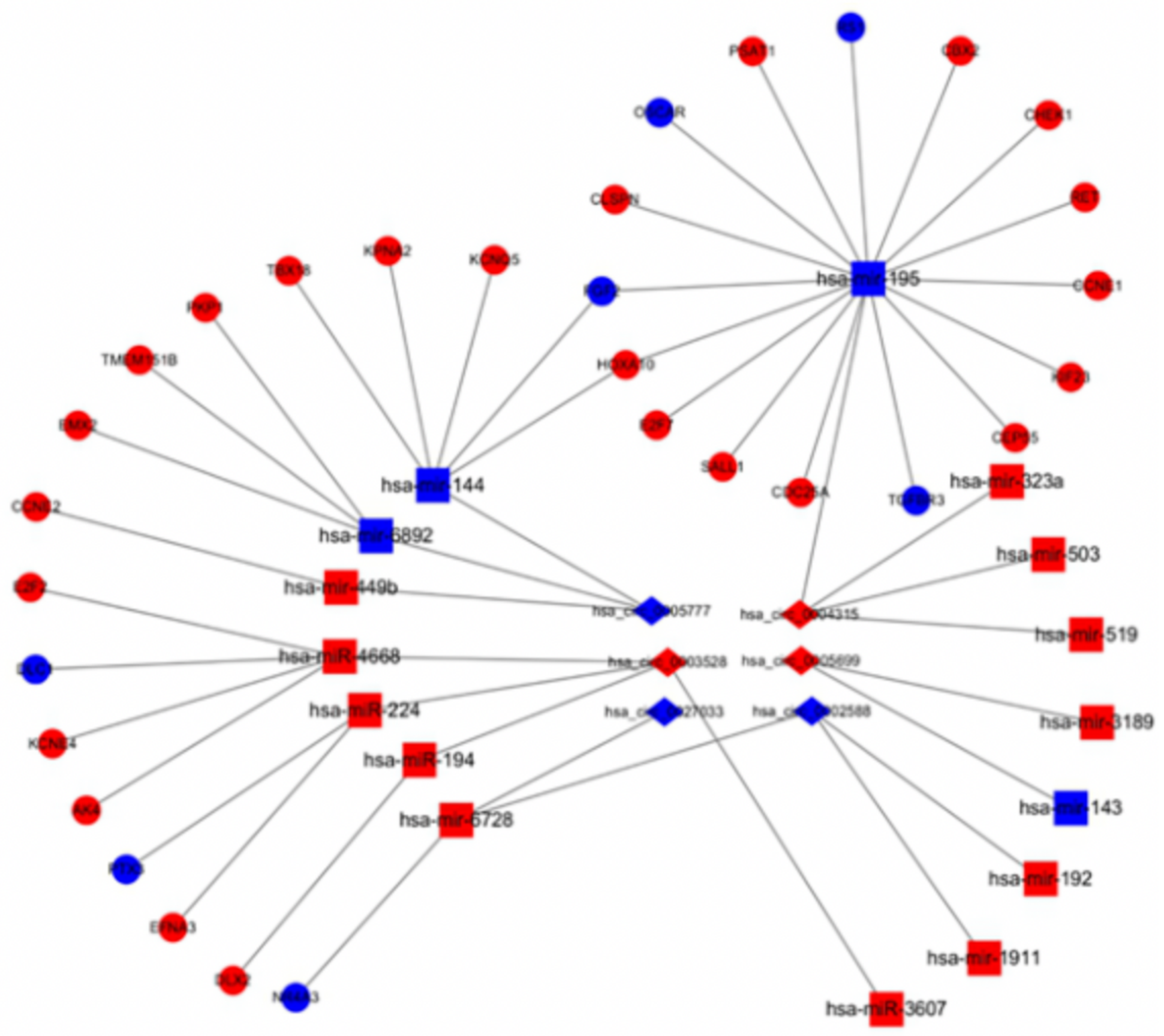

Figure 4

The ceRNA network of circRNA-miRNA-mRNA in LUAC. Diamonds indicate circRNA, square indicate miRNA and round indicate mRNA. The nodes highlighted in red and blue represent up-regulation and down-regulation. 


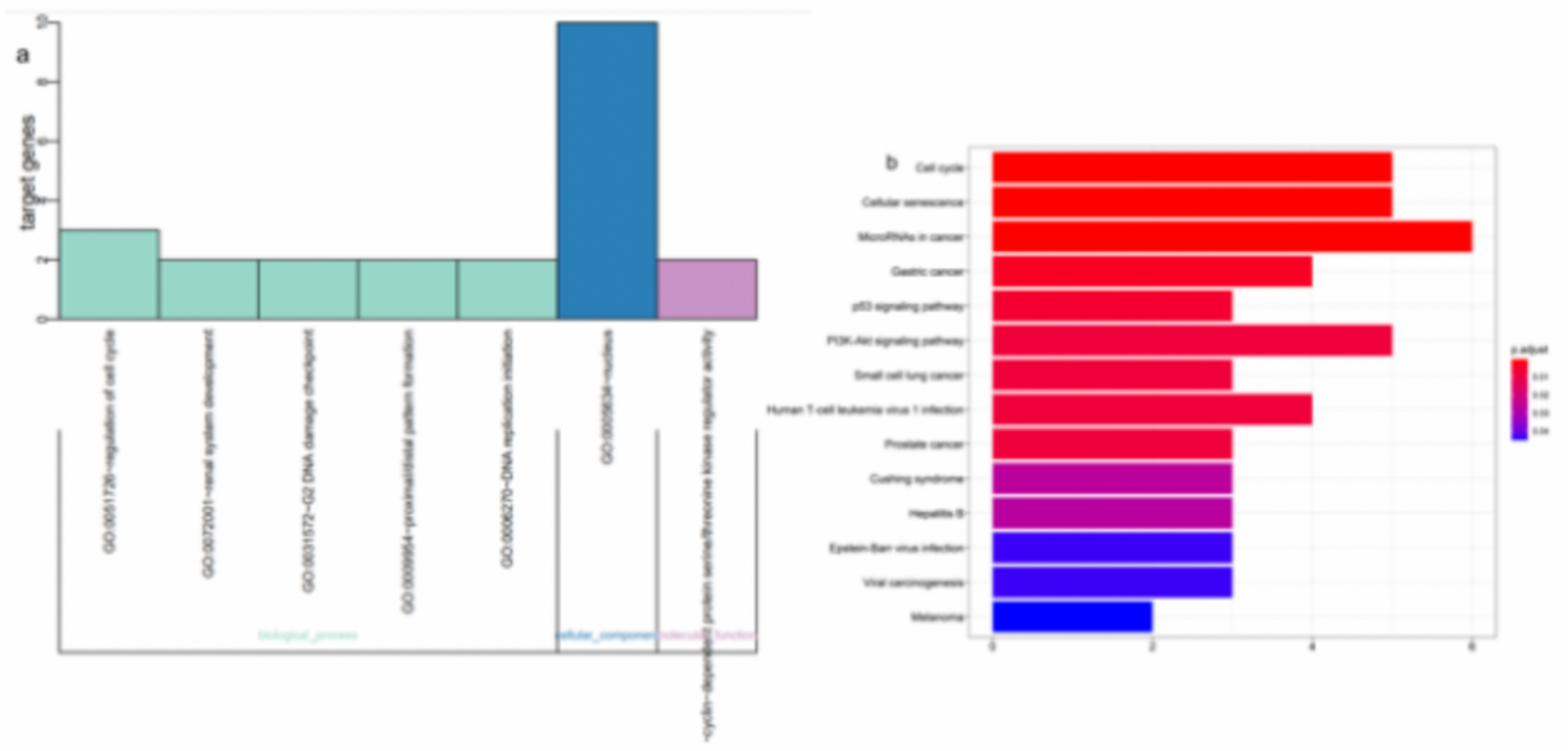

\section{Figure 5}

GO and KEGG functional enrichment analyses of the differentially expressed mRNA in the ceRNA network. a The green represents biological processes; blue represents molecular fuction; purple represents molecular fuction. $b$ The enrichment analysis of the KEGG pathway. A P<0.01 was considered to indicate a significant difference
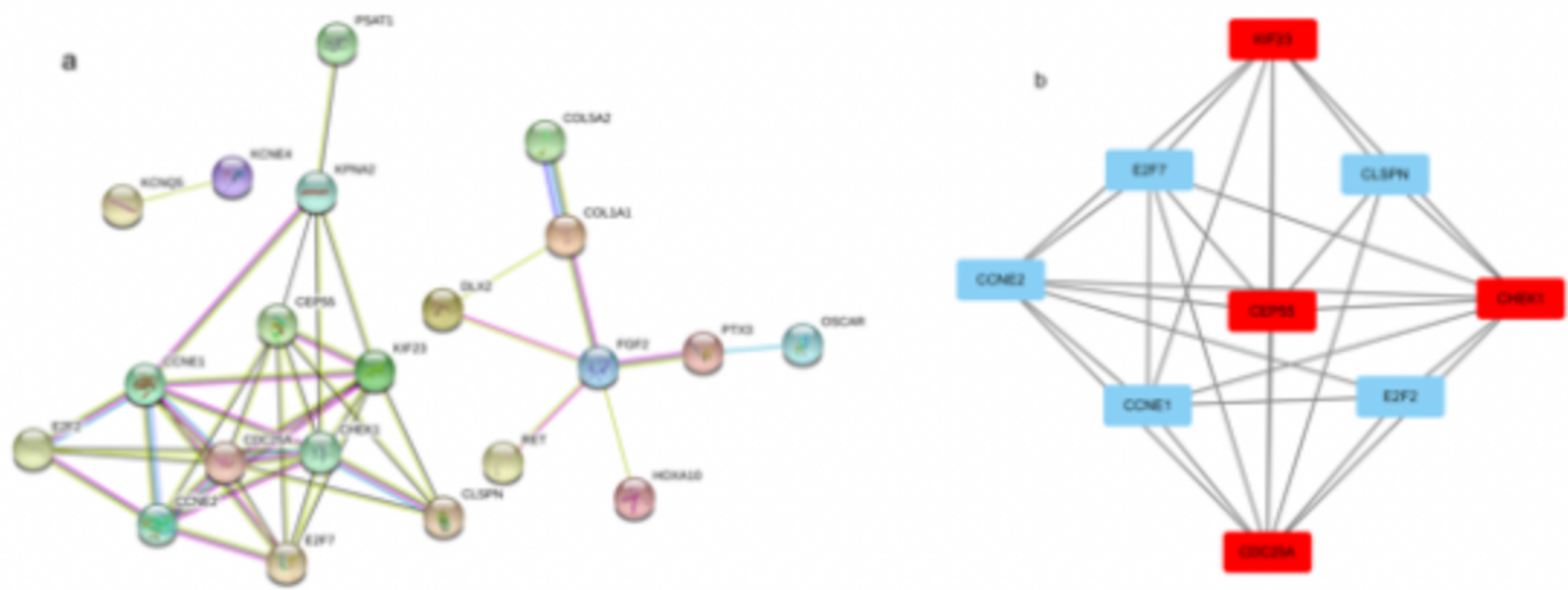

\section{Figure 6}

Identification of hub genes from the PPI network with STRING software and R software. a PPI network of 33 genes. b PPI network of four hub genes which are expressed in red 

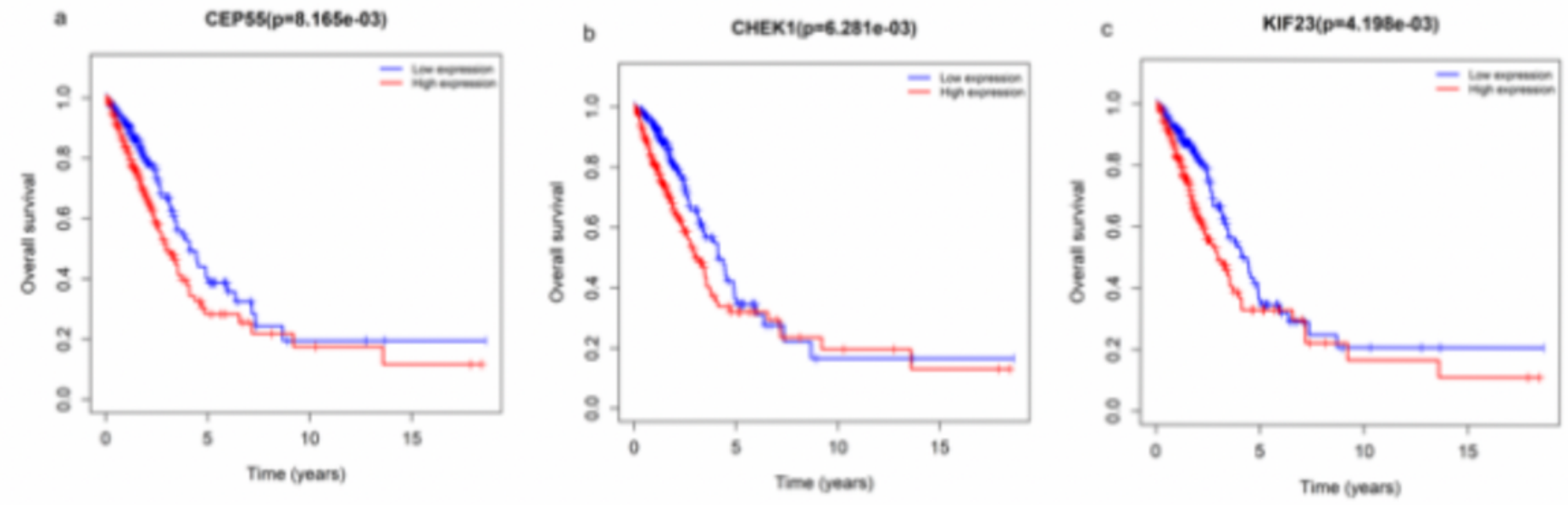

Figure 7

Overall survival of the three hub genes, high expression in red and low expression in blue $(\mathrm{p}<0.05)$. a CEP55, b CHEK1, c KIF 